\title{
Charged particle multiplicities at BRAHMS
}

\author{
R. Debbe for the BRAHMS collaboration
}

\begin{abstract}
This report presents the measurement of charged particle multiplicity densities $d N / d \eta$ in ultrarelativistic heavy ion collisions as function of $\eta$ and the centrality of the collisions. This distributions were extracted from data collected by the BRAHMS collaboration during the first RHIC run with gold ions at $\sqrt{s_{N N}}=130 \mathrm{~A} \cdot \mathrm{GeV}$. The analysis method is described and, results are compared to some model predictions.
\end{abstract}

\section{INTRODUCTION}

Last year's RHIC run opened a new frontier in the study of nuclear matter at extreme energy and baryon number densities. With the accelerator running at $70 \%$ of its design energy $\left(\sqrt{s_{N N}}=130 \mathrm{~A} \cdot \mathrm{GeV}\right)$ all four experiments collected data that is producing interesting results. In this report we describe the measurement of the charged particle multiplicity densities in pseudo-rapidity space obtained with the global detectors of the BRAHMS experiment.

Within the frame of some theoretical models the pseudo-rapidity distribution of charged particle densities $d N / d \eta$ offers a view to the earliest and latest stages of the collision evolution. Present understanding of high energy heavy-ion interactions starts with the two ions highly Lorentz contracted going through each other. The time scale of this collision is hypothesized to last $1 \mathrm{fm} / \mathrm{c}$ [1]. From previous measurements at lower energies it was expected that the baryons from the beam would have become practically transparent to each other such that after the collision no net baryon number would be found at mid-rapidity and the baryons would be found 1 or 2 units of rapidity lower than the beam rapidity value (5). From the first results of last years run, it is becoming evident that the process is more complicated, the measured anti-baryon to baryon ratio at mid-rapidity is not 1 but 0.66 [2]. Some mechanism is putting baryonic matter at mid-rapidity while the original baryons are expected to remain close to beam rapidity.

A longitudinal as well as transverse expansion has been hypothesized and measured at different energy regimes as well as at RHIC $[3,4,5]$. The exact nature of this expansion is not fully understood but hydrodynamic models do reproduce the data.

After $\sim 10 \mathrm{fm} / \mathrm{c}$ the expansion reaches a point where the particles become so far apart that interactions among them ceases, and from there on, the particles stream toward the detectors inempeded (expect from interactions with matter in beam pipes or the detectors themselves). This stage of the expansion is referred as the freeze out stage and because the interaction between particles can be an exchange of momentum or reactions producing other particles the freeze out stage can be thermal or what is know as chemical freeze out respectively. Fits to measured momentum distributions and particle abundances indicate that thermal freeze out is the latest stage of the system.

Multiplicity density $d N / d \eta$ is related to the entropy density $d S / d \eta[1]$, and hydrodynamical expansion proceeds at constant entropy. If the system actually expands like a fluid, the measured multiplicity densities can be used to set limits to the entropy density produced during initial stages of the collision.

The shape of the multiplicity distribution is also a window to later stages of the evolution of the system, as particles are transported to different rapidities by secondary interactions.

\section{DESCRIPTION OF THE EXPERIMENT}

The BRAHMS experiment consists of two small acceptance spectrometers; the MRS spectrometer measures particles produced at mid-rapidity and can rotate from 90 to 32 degrees, and the FS spectrometer that measures particles close to the beam line, this spectrometer can be placed as close as 2.3 degrees with respect to the beam line and can rotate to bigger angles up to 30 degrees. More detailed description of both spectrometers can be found in [6]. 
Two systems are used to identify the collisions and to trigger the readout of the information collected by each detector; a pair of Zero Degrees Calorimeters ZDC placed 18 meters away from the nominal interaction point, and two arrays of fast Cerenkov counters (BBC) placed around the beam pipe at $2.15 \mathrm{~m}$ from the nominal IP.

The ZDC detectors consist each of three identical modules made of alternating layers of tungsten and quartz fibers arranged in ribbons and placed at zero degrees. The location of these detectors allows the detection of neutrons emitted from the interactions at small angles with respect to the beam direction $|\theta|<2 \mathrm{mrad}$. The time resolution of these calorimeters is as good as to define the vertex location within $\sim 3.6 \mathrm{~cm}$. This system provides the least biased trigger for the experiment because it is particularly well designed to detect neutrons produced by Coulomb interaction between the gold ions in very peripheral interactions.

The BBC system is made of two sets of Cherenkov radiators seen by fast photo-multipliers. Each array has an angular coverage of $2.5 \leq|\eta| \leq 4.7$. These detectors have an intrinsic time resolution of $65 \mathrm{ps}$ and are used to define the time zero of the collision, the location of the vertex along the beam axis, and they also provide information about the multiplicity of particles within their angular coverage. During the first RHIC run the second trigger used for the data acquisition was made of a narrow time coincidence of signal from one PMT from each array. This trigger is more biased than the one defined with the ZDC, but selects well defined nuclear collisions.

The centrality of the collision is measured with the multiplicity array (MA). This detector has two components: an array of scintillator tiles called TMA, and a silicon strip array (SiMA) with finer segmentation. The scintillator tiles are $12 \mathrm{~cm}$ squares and $5 \mathrm{~mm}$ thick. A complete description of this detector is being prepared for publication [12]. The plastic tiles are mounted in a hexagonal barrel at $13.9 \mathrm{~cm}$ from the beam axis in groups of 8 . Tiles that would obstruct the path of particles into the MRS or FFS spectrometers were removed. The total number of tiles in these detector is 38 and their nominal angular coverage is $-3<\eta<3$. The SiMA detector consist of $25 \mathrm{Si}$ wafers $4 \mathrm{~cm} \mathrm{X6} \mathrm{cm} X 300$ $\mu \mathrm{m}$ subdivided in seven strips with a pitch of $0.86 \mathrm{~cm}$. These detectors are mounted $5.3 \mathrm{~cm}$ away from the beam axis in a hexagonal barrel in similar way as the TMA.

The first TPC of the MRS spectrometer called TPM1, is also used to evaluate the multiplicity of the event. This detector consists of a volume of gas $(90 \%$ argon and $10 \% \mathrm{CO} 2)(\mathrm{w}, \mathrm{h}, \mathrm{d})=37.5 \mathrm{~cm}, 21.0 \mathrm{~cm}, 36.0 \mathrm{~cm})$. The center of TPM1 is located $94.5 \mathrm{~cm}$ from the nominal vertex. This detector is the preferred vertex reconstructor; events with two or more tracks pointing to the beam axis provide a vertex $\mathrm{z}$ coordinate with a resolutions of $0.5 \mathrm{~cm}$.

During last year's run the MRS spectrometer was placed at $40^{\circ}, 60^{\circ}$, and $90^{\circ}$ corresponding to $\eta=1.1,0.55$, and 0.0 respectively. A schematic of the experimental setup near the IP is shown in the upper left insert of figure 1 .

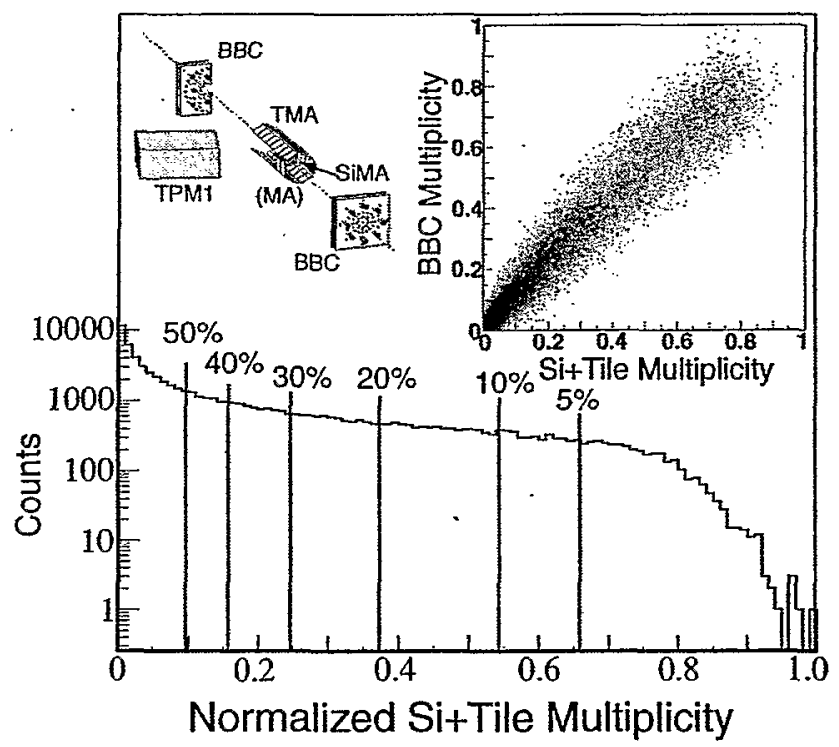

FIGURE 1. Figure 1. Normalized Multiplicity distribution extracted with the MA counter. Upper left insert shows a schematic of the detector setup used in the analysis presented in this report. The upper right insert shows the good correlation between normalized multiplicities extracted from $\mathrm{BBC}$ and $\mathrm{MA}$ respectively. 

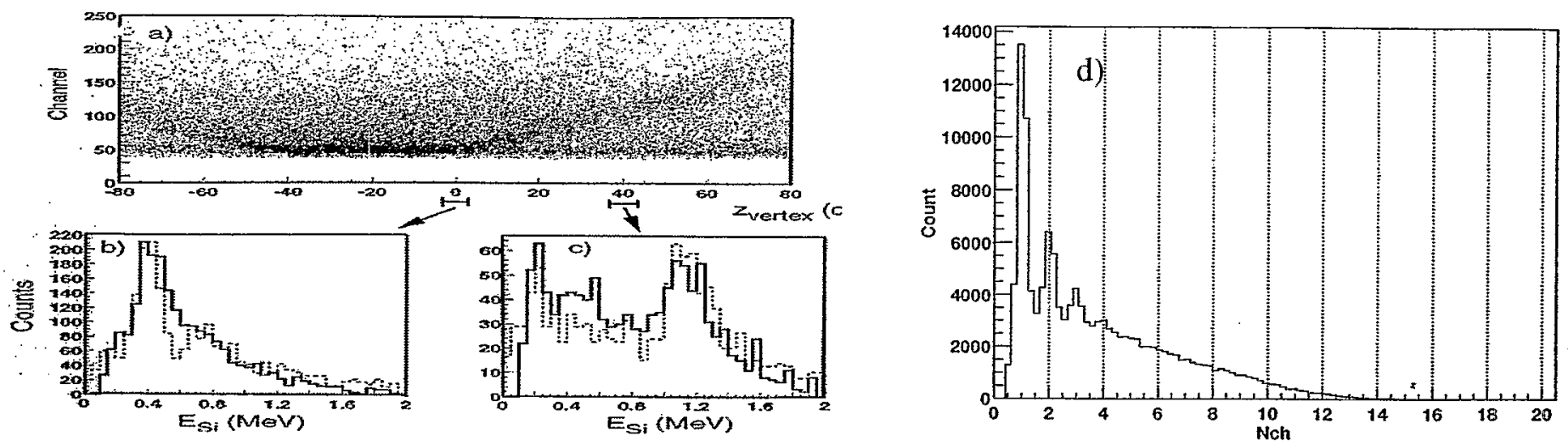

FIGURE 2. Energy deposited in one of the silicon detectors versus vertex position. A band related to a single minimum-ionizing particle is clearly seen in panel a. Bottom panels show the matching of data and GEANT simulated single MIP peaks used for energy calibration of the detector. Similar work is done to calibrate the TMA. Panel d shows the ADC distribution of one of the $\mathrm{BBC}$ detectors. The pedestal of the distribution has been subtracted and up to three MIPs can clearly be seen.

\section{DATA ANALYSIS}

The centrality of the collision is directly related to its charged particle multiplicity, BRAHMS measures the centrality of each collision as a fraction of a minimum-biased multiplicity distribution.

The first stage in the analysis of each event is the extraction of the charged particle multiplicity. That multiplicity can be extracted from the MA or the BBC detectors depending on the location of the interaction vertex. If the vertex is confined to the physical size of the TMA ( $30 \mathrm{~cm}$ from the center of the array), the multiplicity of the event will be the average of the values measured with the TMA and SiMA detectors.

The minimum-biased multiplicity distribution extracted from the MA counters requires that there was a time coincidence between the two ZDC detectors, the vertex of the collision be within the limits mentioned above, and a multiplicity of at least four in the TMA array. Simulations based in the event generator HIJNG and a GEANT package that includes all elements of the experimental setup, indicate that this minimum-biased distribution represents $95 \%$ of the total nuclear cross section.

If the vertex of the collision lies beyond the physical size of the MA counter, (vertices within $120 \mathrm{~cm}$ of the MA center) the multiplicity of the event is extracted from the number of charged particles that impinged on the BBC detectors. This in turn, extends the coverage of the $B B C$ detectors up to $\eta=4.6$ Figure 1 shows the minimum-biased multiplicity distribution extracted with the MA

The upper right insert in figure 1 shows the good correlation between multiplicities extracted from the MA as well as the BBC counters. In the angular region where both system have overlapping coverage; $3.0 \leq \eta \leq 4.2$ the BBC data was analyzed with both centrality selections and the results came up within $1 \%$ of each other.

The procedure to extract multiplicities from both of the MA systems is the same, the vertex of the collision is measured with the TPC, BBC or ZDC, an average angle of incidence to tiles or strips is calculated from that point and the surveyed position of each detector element. Tiles and strips are for the remainder of the analysis grouped in rings of equal $\eta$. A factor to translate the amount of energy deposited in each element to the number of primary charged particles is extracted from a GEANT simulation. A sample of the $6 \%$ most central events from an event generator is used as input, the mean value of the number of primary charged particles hitting each element is extracted, as well as the mean energy deposited. The energy deposited includes secondary interactions and different angles of incidence as well as different particle mixtures. This simulation is done for different vertex positions and a correlation between number of primary particles and energy deposited is obtained. The amount of charge collected in each element of the array is calibrated by matching the data and the Monte-Carlo simulations in very peripheral events where a peak assigned to single minimum-ionizing-particle (MIP) is clearly visible. Figure 2 a shows the band of 1 MIP in the silicon array and its correlation to the GEANT simulated distributions appears in panels $b$ and $c$.

To extract the pseudo-rapidity distributions at very forward angle we use the information from the Beam-Beam detectors, as can be seen in figure $2 \mathrm{~d}$, these detectors are self calibrated and have few charged particles going through 
them. The first step to the analysis consist on the calculation of the $\mathrm{z}$ coordinate of the collision vertex.

Once the vertex is known, each element of the Beam-Beam array is assigned a value of $\eta$ and $\Delta \eta$. The amount of charge collected on each ADC is pedestal subtracted and calibrated using the peaks of the distribution. The result is now a count of charged particles that impinged on that particular detector. For the analysis of this system the detectors are organized in rings of equal $\eta$, and once the count of charged particles is done, a geometrical acceptance for each ring is calculated and a distribution of multiplicity in $\eta$ space is produced. The contribution from secondary interaction is done with a full simulation of the experiment within the GEANT framework.

The TPM1 TPC is also used to extract one value of $d N / d \eta$ at each angle of the spectrometer. The analysis in this case consists in the counting of tracks that point to a well defined point along the beam line. Contributions from background tracks are eliminated by cuts to the y coordinate of the projection to the nominal beam position. The number of tracks is then corrected for geometrical acceptance and recostrunction efficiencies obtained by embedding simulated tracks in real events. At very central events the agreement between results from all three system is remarkable.

The main contribution to the systematic uncertainty in the TMA lies in the calibration of the energy deposited. The analysis relies on only one measured point (single MIP), but the most central events may have up to one hundred hits per tile. The linearity of the photo-multipliers was measured in the laboratory, and it was found to be within manufacturer specification for even the most central events. A second contribution to a systematic error in this detector comes from the fact that the contribution from secondary interactions is only as good as the Monte-Carlo simulations. Because this contribution is angle dependent we assign a systematic uncertainty of $\pm 12 \%$ for $\eta<1.5$ increasing up to $\pm 15 \%$ for $\eta \geq 2.0$

The silicon array SiMa has similar systematic uncertainties but they differ in magnitude from the ones assigned to the plastic detectors because they are less massive and have an smaller contribution from interactions in the active volume; the SiMa array has an assigned systematic error of $\pm 8 \%$ for $\eta<1.5$ increasing to $\pm 10 \%$ for $\eta \geq 2.5$

The BBC counters have an assigned overall systematic error of $\pm 10 \%$ that includes secondary interactions estimated with Monte-Carlo techniques, and corrections to non-linearity of the photo-multipliers response. The results obtained with the TPC TPM1 have an assigned error of $+9 \% /-7 \%$ for the most central events and $\pm 6 \%$ for the most peripheral.

\section{COMPARISON TO MODELS}

Figure 4 shows charged particle pseudo-rapidity densities for four different centrality cuts. The $d N / d \eta$ distributions have been symmetrized and are uncertainty weighted averages of the results obtained with the MA, BBC and TPM1 detectors. Starting with the first results out of the RICH 2000 run presented by the PHOBOS collaboration [7], and latter reports from the other three experiments, it was clear that the majority of models in the field predicted much higher production of charged particles A summary of the comparison of data and models at QM2001 can be reviewed in [8]. For the present report we concentrate on the models that came closer to the actual particle densities in $\mathrm{Au}-\mathrm{Au}$ collisions. The curves are obtained with two models; HIING [9] and AMPT [10] subjected to the same cuts as the data. HIJNG is an event generator developed to study jet and multiparticle production in high energy pp and AA collisions. It emphasizes the applicability of perturbative QCD in semi-hard parton scatterings that are expected to be of importance at RHIC energies. It also takes into account nuclear effects such as parton shadowing and jet quenching. The full line curve in figure 4 was produced with HIJING. Each panel in the figure has data from a determined range of centrality as indicated on the top left corner. For the two most central samples the model reproduces very well the multiplicity density at $\eta=0$ and has lower densities at the peripheral samples. HIJNG does not reproduce well the data at higher values of $\eta$. The second model, AMPT uses HIJNG as a generator of its initial condition and then cascades the partons and the hadrons after hadronization. This model is the one that reproduces the data all across our acceptance.

\section{SUMMARY}

The multiplicity of charged particles as well as its density in $\eta$ has been measured in Au-Au collisions at $\sqrt{s_{N N}}=$ $130 \mathrm{~A} \cdot \mathrm{GeV}$. The data was presented as function of the centrality of the reaction defined as fractions of a minimum biased multiplicity distribution. The data is well reproduced by a parton cascade model setting the initial conditions for a second cascade package that includes interactions between partons and later between hadrons. Results from this analysis have been submitted for publication [11]. 

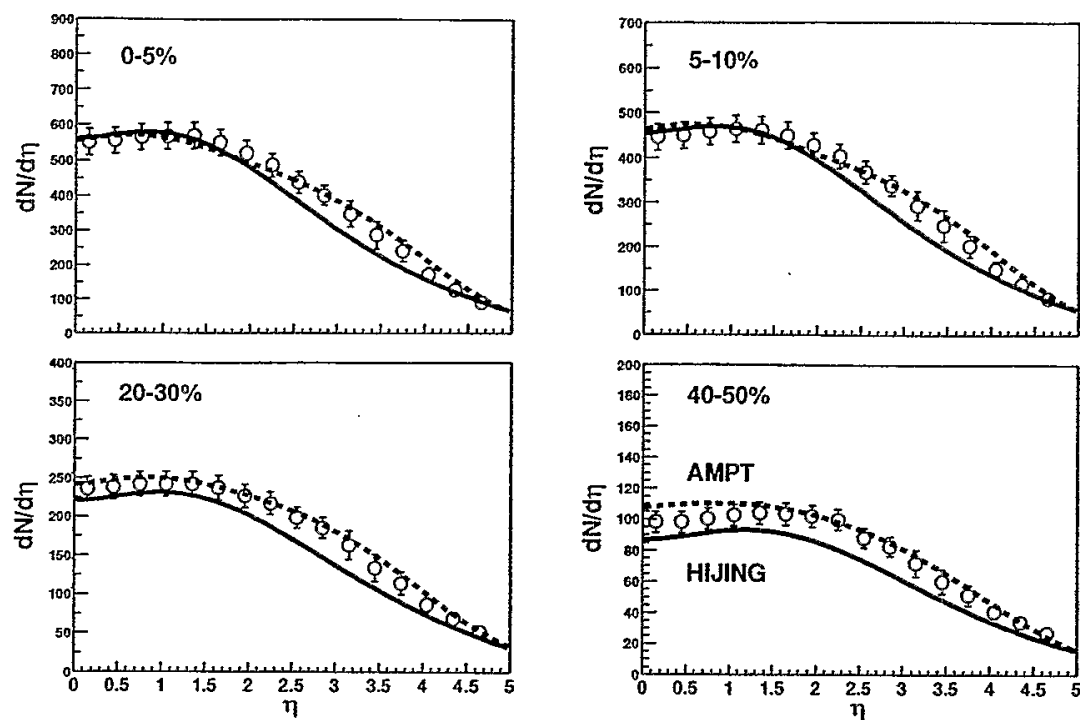

FIGURE 3. BRAHMS Multiplicity density distributions in $\eta$ extracted from four data samples with different centrality selection, compared to model predictions

\section{ACKNOWLEDGMENTS}

This work was supported by the Division of Nuclear Physics of the Office of Science of the U.S. Department of Energy under contracts DE-AC02-98-CH10886, DE-FG03-93-ER40773, DE-FG03-96-ER40981, and DE-FG02-99ER41121, the Danish Natural Science Research Council, the Research Council of Norway, the Jagiellonian University Grants, the Korea Research Foundation Grant, and the Romanian Ministry of Education and Research (5003/1999, 6077/2000).

\section{REFERENCES}

1. J. D. Bjorken, Phys. Rev. D 27 (1983) 140.

2. I. Bearden et al. Phys. Rev. Lett. 87 (2001) 112305.

3. K. H. Ackermann et al. Phys. Rev. Lett. $86402-407$ (2001).

4. E. V. Shuryak Nucl. Phys A638 207-218 (1998).

5. J. Y. Ollitrault Nucl. Phys. A638 195-206 (1998).

6. I. Bearden et al. NIM to be published.

7. B. B. Back et al. Phys. Rev. Lett. 85 (2000) 3100; hep-ex/0007036

8. K. J. Eskola QM2001 Proceedings to be published.

9. X. Wang and M. Gyulassy, Phys. Rev. D 44 (1991) 3501: code HIJING 1.36

10. Bin Zhang, C. M. Ko, Bao-An Li, and Ziwei Lin, Phys. Rev. C 61 (2001) 067901.

11. I. Bearden et al. nucl-ex/0108016.

12. Y.K. Lee et al. to be published NIM A. 
